\title{
Movement signal choreography unaffected by receiver distance in the Australian Jacky lizard, Amphibolurus muricatus
}

\author{
Richard A. Peters • Simon J. Allen
}

Received: 9 December 2008 /Revised: 2 February 2009 /Accepted: 9 March 2009 /Published online: 24 March 2009

(C) Springer-Verlag 2009

\begin{abstract}
Theory explains the structure of animal signals in the context of the receiver sensory systems, the environment through which signals travel and their information content. The influence of signalling context on movementbased signalling strategies is becoming clearer. Building upon recent findings that demonstrated changing environmental plant motion conditions resulted in a change of signalling strategy by the Australian lizard Amphibolurus muricatus, we examined whether receiver distance also influences signalling strategies. We found that signalling lizards did not modify their introductory tail flicking in response to distant viewers in the absence of competing, irrelevant plant image motion despite significant reductions in signal structure at the eye of the viewer. The magnitude of resultant effect sizes strongly suggests that receiver distance does not contribute to signalling strategies as much as the presence of motion noise in the environment.
\end{abstract}

Keywords Amphibolurus muricatus · Jacky lizard · Movement-based signal $\cdot$ Receiver distance .

Signal evolution $\cdot$ Signal structure

Communicated by S. Downes.

R. A. Peters $(\square) \cdot$ S. J. Allen

Centre for Visual Sciences,

Research School of Biological Sciences,

The Australian National University, P.O. Box 475,

Canberra, Australian Capital Territory 2601, Australia

e-mail: richard.peters@anu.edu.au

S. J. Allen

Murdoch University Cetacean Research Unit,

Centre for Fish and Fisheries Research,

Murdoch University, South St.,

Murdoch, Western Australia 6150, Australia

\section{Introduction}

Regardless of modality, the perception of signals varies as distance between the signaller and receiver increases (Dusenbery 1992; Bradbury and Vehrencamp 1998). Distance effects have been most clearly demonstrated for bio-acoustic signals. For instance, sound waves are subjected to predictable levels of attenuation in open habitats (Marten and Marler 1977; Marten et al. 1977; Wiley and Richards 1982), while scattering, reflections off boundaries and other transmission errors, such as reverberations in cluttered environments (e.g. Richards and Wiley 1980; Römer 1993), are likely to be compounded by greater transmission distances. Signalling in the visual modality suffers from many of the same challenges as sound transmission (attenuation and pattern loss), as well as being particularly affected by the presence of occluding objects. The signalling context, therefore, is also likely to influence the reliable transmission of movement-based visual signals.

There is now convincing evidence that some animals are aware of the potential limitations of certain signals and adopt others that are better suited to a given environment and the required transmission distances (e.g. Couldridge and van Staaden 2004). Some primates, for example, use different vocalisations depending on how far away the listeners are likely to be (longtailed macaques, Macaca fascicularis, Palombit 1992; pygmy marmosets, Cebuella pygmaea, de la Torre and Snowdon 2002). An alternative strategy is to alter the structure of a given signal to compensate for greater receiver distances. For example, male zebra finches, Teaniopygia guttata, increase the amplitude of courtship vocalisations for longer range signalling (Brumm and Slater 2006), while male green frogs, Rana clamitans, 
modulate the rate, frequency and duration of their acoustic calls (Owen and Gordon 2005). Comparatively little attention has been paid to the context in which movement-based signals are performed. However, recent findings have revealed the importance for movement-based signalling strategies of habitat light conditions and the spectral qualities of body structures (Leal and Fleishman 2002; Uy and Endler 2004), as well as environmental motion noise (Ord et al. 2007; Peters et al. 2007; Ord and Stamps 2008).

Signalling with movement to distant receivers is predicted to benefit from structural modifications that ensure reliable transmission and detection (e.g. Bradbury and Vehrencamp 1998). There are, however, only two recent studies suggesting that receiver distances influence movement-based signalling strategies. How et al. (2008) described in quantitative detail how fiddler crabs Uca perplexa alter both the intensity and the form of their courtship display with respect to receiver distance. Males displaying to distant females signal for longer and generate claw trajectories with larger horizontal components when compared with signalling to nearby females. Adjustments to signal structure in this case are likely to be necessary for overcoming the sensory limitations in the spatial resolution of crab compound eyes. Further, since claw waving plays a role in mate choice, adjustments to signal structure likely ensures females can reliably assess signaller quality at greater distances.

Receiver distance may also influence the signalling strategies of lizards (Stamps and Barlow 1973). Indeed, receiver distance was found to be a significant predictor of whether or not common anoles, Anolis gundlachi, include a conspicuous alerting component, comprising rapid four-legged pushups (Ord and Stamps 2008). The authors further demonstrated that the facultative addition of this component to the typical display enhances signal detection by viewers. It is not known whether the provision of additional 'information' assists with opponent assessment; however, the lizards' behaviour strongly suggests that a sensory constraint affecting reliable detection is likely. It is unclear from this study whether the lizards adjusted their signalling strategies directly in response to receiver distances, as variation in other environmental factors, such as habitat light conditions and the level of environmental motion noise, also significantly predicted the addition of the alerting component (Ord and Stamps 2008).

If we are to understand the co-evolution of movement-based signalling strategies and receiver sensory systems in detail, then it is necessary to tease apart the relative contribution of these factors. We do so here by building upon earlier work with the Australian Jacky lizard, Amphibolurus muricatus. During territorial displays, males perform a stereotyped display in response to conspecific intruders that starts with tail flicking and is followed by rapid delivery of four distinct motor patterns (Peters and Ord 2003). The introductory tail flicking alerts receivers to the rest of the display (Peters and Ord 2003), which is believed to reveal individual qualities of the signaller that opponents/intruders assess. We recently demonstrated that $A$. muricatus varied temporal aspects of their tail flicking in calm and windy conditions (Peters et al. 2007). The lizards lengthened the duration of tail flicking and switched to intermittent signalling when viewers were located at a relatively close distance of $1 \mathrm{~m}$. Environmental motion noise therefore exerts a strong effect on the signalling strategies of this lizard. It also demonstrated that lizards are sensitive to the efficacy of their motion signals and are able to make adjustments when necessary.

To what degree tail flicking by $A$. muricatus is influenced by other factors affecting signal transmission is unclear. Our goal for this study was to consider the effect of receiver distance while holding environmental motion noise constant. A. muricatus tail flick regardless of signalling context so we looked for variation in aspects of tail flick structure. Tail morphology allows for tremendous variation in signalling dynamics, from small amplitude flicks involving only the tip of the tail through to large amplitude, whole tail movements that sweep three-dimensional space. We predicted that lizards might adjust signalling if they do indeed use small amplitudes for close signaller-receiver distances. The use of minimal movements at close distances would likely reduce signalling costs, particularly in terms of increased visibility to predators. At greater distances, however, small amplitudes may be difficult to resolve due to limits in spatial acuity. This would be particularly pertinent if the signal was seen in the receiver's peripheral vision where ganglion cell densities decline and acuity is reduced (New 2008). We firstly examined the temporal dynamics of tail flicking (duration, time in motion) as a direct follow-on from our motion noise experiment. The results described above and elsewhere (e.g. Bradbury and Vehrencamp 1998) suggested that increasing display amplitude and/or speed can ensure detection from further away. Accordingly, we also considered changes to the angular extent and angular speed of tail movements. Importantly, we adopted the same experimental design as the aforementioned plant motion study (Peters et al. 2007). We subsequently compared effect sizes for receiver distance and plant motion to examine their relative influence on the evolution of movement-based signals that have an alerting function. 


\section{Methods}

\section{Subjects}

Sixteen adult male A. muricatus (White ex Shaw 1970) lizards were wild-caught by noosing in December 2007 from National Parks on the south coast of New South Wales, Australia. Lizards were transported in calico bags by air-conditioned car to outdoor holding enclosures at The Australian National University Coastal Campus (The Edith and Joy London Foundation, Kioloa, NSW). Enclosures $(1.2 \times 1.2 \times 0.9 \mathrm{~m})$ were made from galvanised metal sheets with branches for basking and vegetation for cover. Crickets (Acheta domesticus) were provided and water was available from a small bowl. Lizards were housed individually in holding enclosures for between 4 and 9 weeks before being transferred to a testing enclosure. There, they were allowed to settle, visually isolated from other lizards, for 1 to 2 weeks before testing. Lizards were kept in captivity for a maximum of 4 months before being released at the site of capture, again transported as before.

Experimental design and procedure

Our aim was to examine the effect of receiver distance on introductory tail flicking. In Peters et al. 2007, plant motion was found to exert a strong effect on tail flicking strategies. The main finding of longer signal durations in windy conditions was associated with an effect size of $r=0.701$ (95\% confidence interval (CI) $0.430-0.770$; calculated from the original dataset using formula described below). To facilitate comparisons of the relative effect of plant motion and receiver distances, we adopted the same experimental design and statistical approach as Peters et al. (2007).

We constructed four outdoor testing enclosures (Fig. 1) made from galvanised metal sheets (sides, back), galvanised wire (top) and Perspex (front). All enclosures were set up in the same way, with branches for basking located centrally within the enclosures. No plant material was provided in the test enclosures to ensure lizards did not make signalling adjustments in response to changes in the movement patterns of wind-blown plants. Plywood dividers were placed in front of the enclosures to prevent focal lizards (residents) from seeing 'intruders' outside of their own testing session. The conspecific male intruders were presented to residents in custom-made portable reptile enclosures $(1.2 \times 0.4 \times 0.6 \mathrm{~m})$ made from melamine coated particle board (back, sides), galvanised wire (top) and Perspex (front). Each stimulus (intruder) enclosure featured a natural substrate (sand-soil mixture) and branches for basking. A small video camera was positioned centrally at the top of the stimulus enclosure to film resident lizard responses (Fig. 1). As the stimulus enclosures were portable, we were able to shift their physical position to vary the resident-intruder distance from 1 (near) to $3.5 \mathrm{~m}$ (far; Fig. 1).

We tested 16 lizards in blocks of four during January and February 2008. Half of the lizards were presented first with a near intruder followed by a far intruder on the following day; the remaining lizards experienced far intruders first. Intruder pairings (near and far) presented to residents were approximately matched in size and presented at similar
Fig. 1 Schematic diagram of the experimental setup showing the resident and intruder enclosures. Branches for basking were located centrally in the resident enclosures (star). Plywood dividers (dashed lines) were positioned in front of the resident enclosures to ensure only the focal lizard could see the intruder during each testing session. Intruder enclosures were physically moved between the near $(1 \mathrm{~m})$ and far $(3.5 \mathrm{~m})$ distances. A camera was positioned on top of the intruder enclosure to film residents

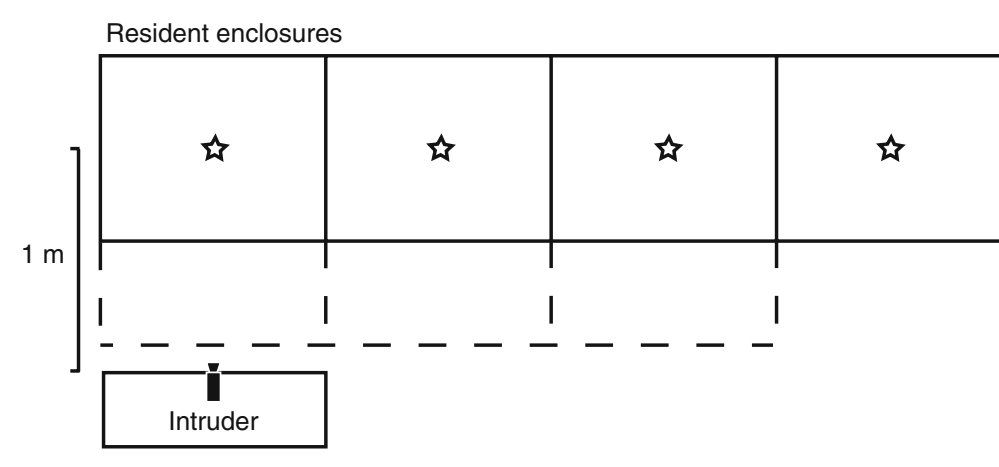

$3.5 \mathrm{~m}$ 
times of the day. All testing was conducted between 1000 and 1400 hours on clear sunny days when natural conditions encouraged basking. At the start of the day, conspecific intruder males were placed in the stimulus enclosures and allowed to settle for at least $1 \mathrm{~h}$ prior to testing. At the start of each session, the video recorder was set to record and the plywood divider in front of the testing enclosure was lowered so that the focal resident and intruder became visible to each other. The session terminated after the first display bout by the resident focal lizard, at which time the plywood divider was returned to its position and recording stopped.

Data analysis and statistical procedures

We restricted our analyses to the 11 lizards that performed territorial displays in response to intruders at both near and far distances. The excluded lizards either did not perform territorial displays in the first session or did so in the first session only ( $n=4$ and 1 , respectively). Video clips were imported into Final Cut Express (Apple Inc.) software for direct analysis and also exported for use by separate software, as discussed below. We considered variation in introductory tail flicking within three categories: temporal, spatial and angular speed changes. We examined the effect of distance separately for each variable within each category using R 2.3.1 (R Development Core Team 2006), transforming the data prior to analysis using $\log _{10}$ or square-root transformations where appropriate. We set up linear mixed effects models ( $\mathrm{R}$ 2.3.1) fitting distance as a fixed factor and including lizard identity and block number as random factors. The statistical significance of individual model parameters was tested with the $F$ test. Models were checked graphically for outliers and appropriate error distributions. We also calculated effect sizes with $95 \%$ confidence intervals for each variable, using formulas appropriate for mixed effects models presented in Nakagawa and Cuthill (2007). To facilitate comparisons with other studies, we report effect sizes as $r$ statistics based on the $t$ values from our mixed effects model (Eq. 24 in Nakagawa and Cuthill 2007).

\section{Temporal considerations}

We logged events in Final Cut Express to quantify the timing of tail flicking to an accuracy of $40 \mathrm{~ms}$ (phase alternating line (PAL) frame rate). Tail flicking can be continuous or intermittent (Fig. 2a). Consequently, we calculated the time from first tail movement to the start of the push-up component of the display ('tail flick duration'), as well as the actual 'time in motion' during tail flicking by subtracting cumulative pause durations from tail flick duration. A final measure was computed from the quotient of time in motion and tail flick duration to indicate the 'relative effort' in tail flicking.

\section{Spatial considerations}

Each session was exported from Final Cut Express as separate Audio Video Interleave (AVI) files and imported into Matlab (MathWorks Inc.). Problems with video artefacts in two clips restricted our analysis to nine subjects. We used custom-written software (Jan Hemmi, The Australian National University) to track multiple positions of the tail over time to consider changes in the angular extent of tail flicking between the two intruder distances. Four features were tracked: the tip, base and two intermediate points (Fig. 2b). The intermediate points were selected to divide the tail into three roughly equal segments and could be tracked in successive frames using natural markings. However, we present data only for the tail tip and the nearest intermediate point as the other two points revealed little variation across conditions. We collapsed across time (Fig. 2c) and identified the spatial extent (maximum-minimum) in the horizontal and vertical planes (Fig. 2d). Spatial coordinates in pixels were converted to millimetres using an object of known size in the frame before converting to visual angles using the tangent formula

$\theta=2 \operatorname{atan}\left(\frac{x}{2 d}\right)$

Here, $x$ is the spatial extent value and $d$ is distance. We first calculated the visual angle from the viewpoint of the receiver using distance values as tested (1 and $3.5 \mathrm{~m}$ ). However, to determine whether signallers adjusted the spatial component of their flicking, we calculated a second set of values in which we used $d$ of $1 \mathrm{~m}$ for both conditions. These measures allow us to consider the signal as it was 'perceived' by the receiver and 'generated' by the signaller. We therefore generated 'perceived' and 'generated' representations of 'horizontal' and 'vertical' visual angles for the 'tip' and 'intermediate' positions.

Signalling speeds

The exported AVI files were also used to calculate the image motion generated by tail flicking to consider whether lizards changed signalling speed. As above, our sample for these calculations was restricted to nine subjects. Image motion was determined using another custom written Matlab program that has been described in detail elsewhere (Peters et al. 2002). Briefly, the program is based on a gradient motion detector model and calculated the velocity field in image sequences based on temporal and spatial derivatives of filtered versions of image intensity (see Peters et al. 2002 and references therein for details). Each 
a

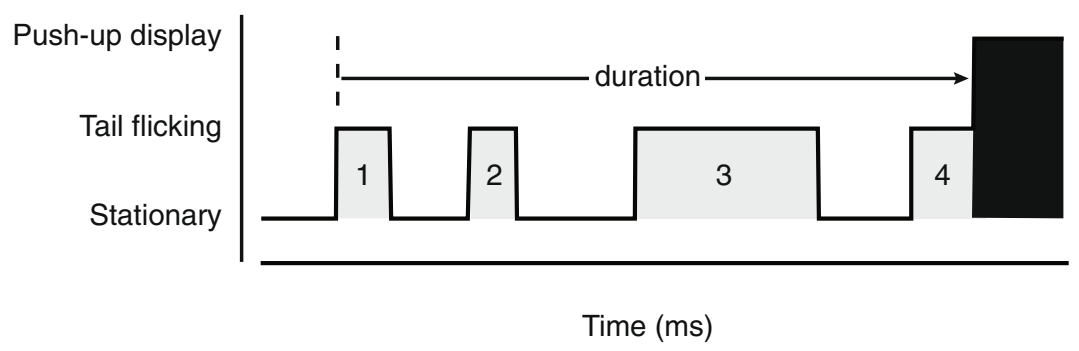

b
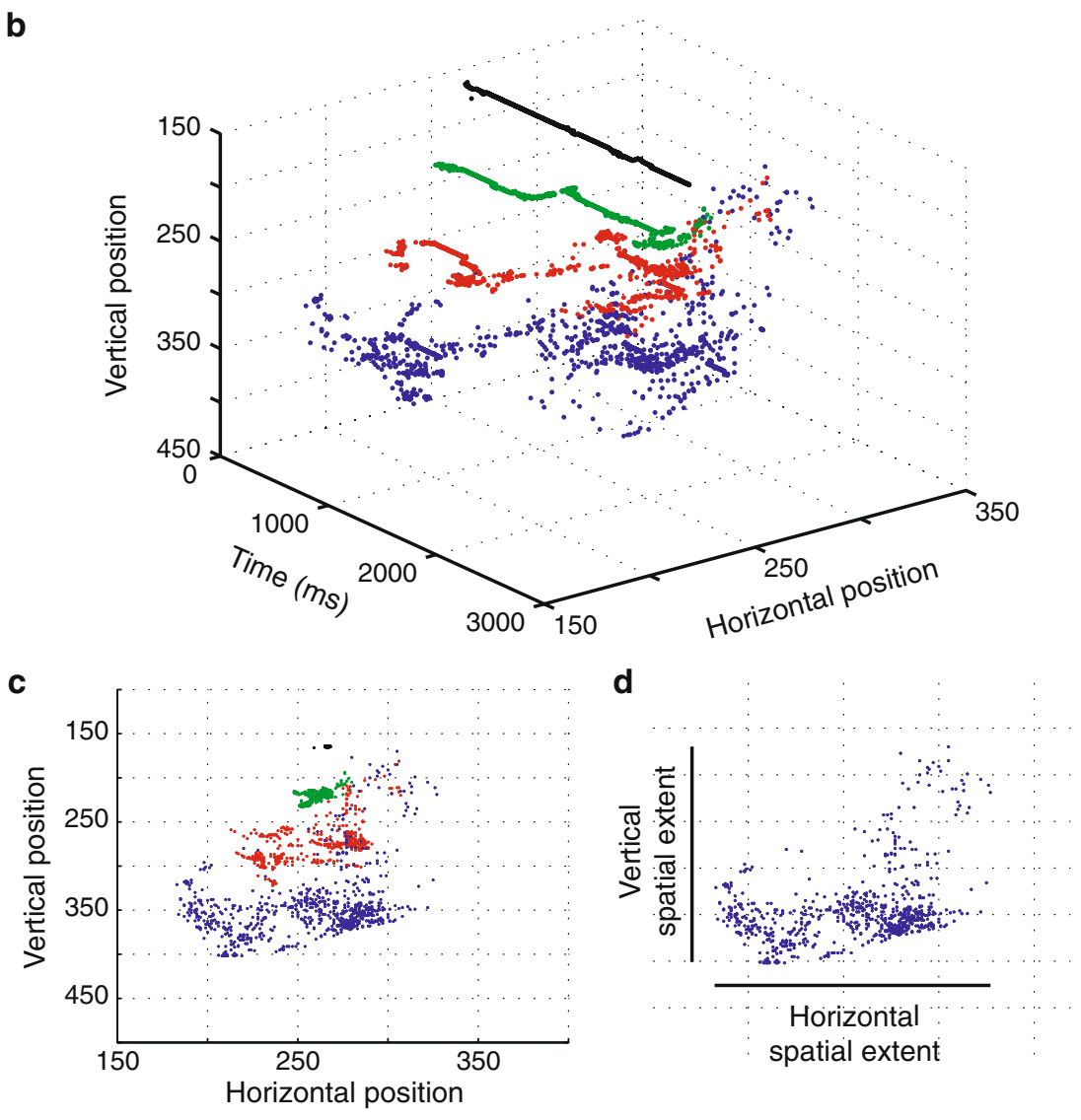

Fig. 2 a Schematic profile of a Jacky lizard tail flick sequence. In this example, the tail starts to move and remains in motion for a short period of time before stopping (shaded region 1). Such intermittent motion is repeated twice more (shaded regions 2 and 3). The tail begins moving for a fourth time (shaded region 4) before the onset of the rest of the display (black region), which is centred on a push-up motor pattern. Here, the arrow from the dotted line to the onset of the display represents tail flick duration. The summation of time for regions $1-4$ gives the time in motion, while signalling effort is computed from the quotient of time in motion and duration. b The position of the tail was tracked over time at four points: the tip, base and two intermediate points that divided the tail into three roughly equal segments and could be tracked in successive frames using

natural markings. In this example, the lizard was perched on a branch above the ground, and the tail flicked between the branch and the ground. The change in position of the base (black circle) of the tail is negligible, as indicated by the relatively straight profile, while the intermediate point nearest to the tail (green circle) shows only modest positional changes. In contrast, considerable changes over time can be seen for the tail tip (blue circle) and intermediate point nearest to the tip (red circle). $\mathbf{c}$ The position data in $\mathbf{b}$ collapsed in the time domain. d To estimate the angular extent of tail flicks, we calculated the spread of position markers in the vertical and horizontal planes by subtracting the lowest/left-most points from the highest/right-most points, respectively. The resultant value was converted to degrees of visual angle as described in the text

image was down-sampled to $144 \times 180$ pixels to reduce computational load. The calculated velocity field comprised the angular speed and direction of movement at each location in the frame. We first extracted the angular speed data and averaged the non-zero data across the image separately for each frame to generate a speed-time profile. In summarising this further, we reasoned that lizards might increase the average speed of movement, or generate a brief 
Fig. 3 Summary data for the temporal considerations of tail flicking produced in response to near (closed square) and far (open square) intruders. a Mean $( \pm \mathrm{SE})$ durations and time spent in motion. $\mathbf{b}$ Mean $( \pm \mathrm{SE})$ values for the relative effort during tail flicking (duration/time in motion) a

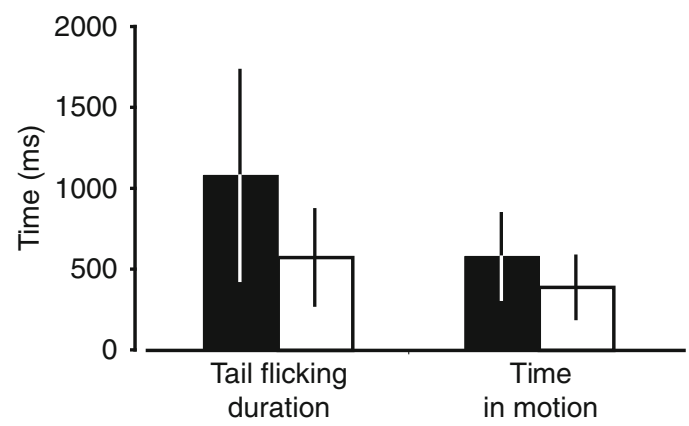

b

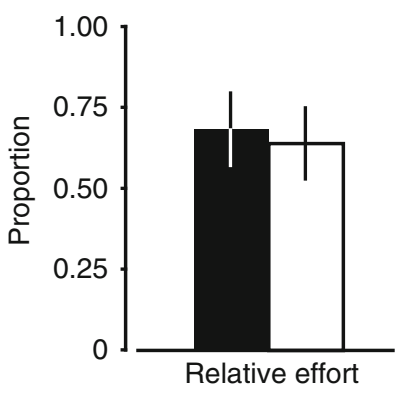

period of faster movement at some point within the sequence. We therefore averaged across the profile to determine the 'average angular speed' as well to identify the 'maximum angular speed' value from the frame sequence. We converted speed measures from pixels/frame to degrees/s using the tangent formula above, estimating speeds that would be perceived by the receiver $(d=1$ and $3.5 \mathrm{~m}$ ), as well as scaling to the same viewing distance $(d=1 \mathrm{~m})$ to consider changes made by the signaller.

\section{Results}

\section{Temporal considerations}

We found no evidence for changes to the temporal profile of introductory tail flicking (Fig. 3). The duration of tail flicking and the actual time in motion (Fig. 3a), as well as the relative signalling effort (Fig. 3b), did not differ between distance conditions (Table 1), with estimated effect sizes less than 0.1 (absolute value) suggesting that these temporal parameters do not change under natural conditions.

\section{Spatial considerations}

At the eye of the intruder, the visual angle subtended by tail flicking reduced significantly from near to far distances when measured at the tail tip (Fig. 4a, b, perceived) and the intermediate point (Fig. 4c, d, perceived) in both horizontal and vertical planes (Table 2). Despite the clear reduction in amplitude, however, signalling lizards did not significantly adjust the angular extent of tail flicking in either plane (Table 1; Fig. 4a-d, generated). Effect sizes for variations at the tail tip indicated a small effect, while those for the intermediate point were a little stronger (Table 1).

\section{Signalling speeds}

There was a significant difference for the average (Fig. 5a, perceived) and maximum (Fig. 5b, perceived) angular speeds as would be seen by the intruder (Table 2).
Table 1 Outcome of linear mixed effects models and corresponding effect sizes as generated by the signaller

\begin{tabular}{|c|c|c|c|c|c|c|}
\hline & \multirow[t]{2}{*}{$F$ statistic } & \multirow[t]{2}{*}{$P$ value } & \multirow[t]{2}{*}{$d f$} & \multicolumn{3}{|c|}{ Effect size } \\
\hline & & & & $r$ & $95 \% \mathrm{CI}$ & \\
\hline \multicolumn{7}{|l|}{ Temporal } \\
\hline Tail flick duration & 0.104 & 0.754 & 1,10 & 0.076 & -0.363 & 0.473 \\
\hline Time in motion & 0.228 & 0.645 & 1,10 & 0.077 & -0.230 & 0.344 \\
\hline Signalling effort & 0.146 & 0.712 & 1,10 & -0.090 & -0.483 & 0.352 \\
\hline \multicolumn{7}{|l|}{ Spatial } \\
\hline Tip_horizontal & 0.078 & 0.789 & 1,8 & -0.074 & -0.511 & 0.412 \\
\hline Tip_vertical & 0.179 & 0.685 & 1,8 & 0.112 & -0.385 & 0.537 \\
\hline Intermediate - horizontal & 0.559 & 0.479 & 1,8 & 0.196 & -0.322 & 0.588 \\
\hline Intermediate - vertical & 1.054 & 0.339 & 1,8 & -0.265 & -0.628 & 0.267 \\
\hline \multicolumn{7}{|l|}{ Angular speed } \\
\hline Maximum & 2.799 & 0.138 & 1,8 & 0.408 & -0.137 & 0.709 \\
\hline Average & 0.179 & 0.685 & 1,8 & 0.112 & -0.386 & 0.537 \\
\hline
\end{tabular}


Fig. 4 Summary data for the spatial considerations of tail flicking produced in response to near (closed square) and far (open square) intruders, shown separately for the perceived signal (distance $=1$ and $3.5 \mathrm{~m}$ ) and the generated signal (distance $=1 \mathrm{~m}$ for both). a, b Mean $( \pm \mathrm{SE})$ visual angle for horizontal and vertical planes of motion measured at the tail tip. c, d Mean ( $\pm \mathrm{SE})$ visual angle for horizontal and vertical planes of motion measured at the intermediate point nearest to the tail tip
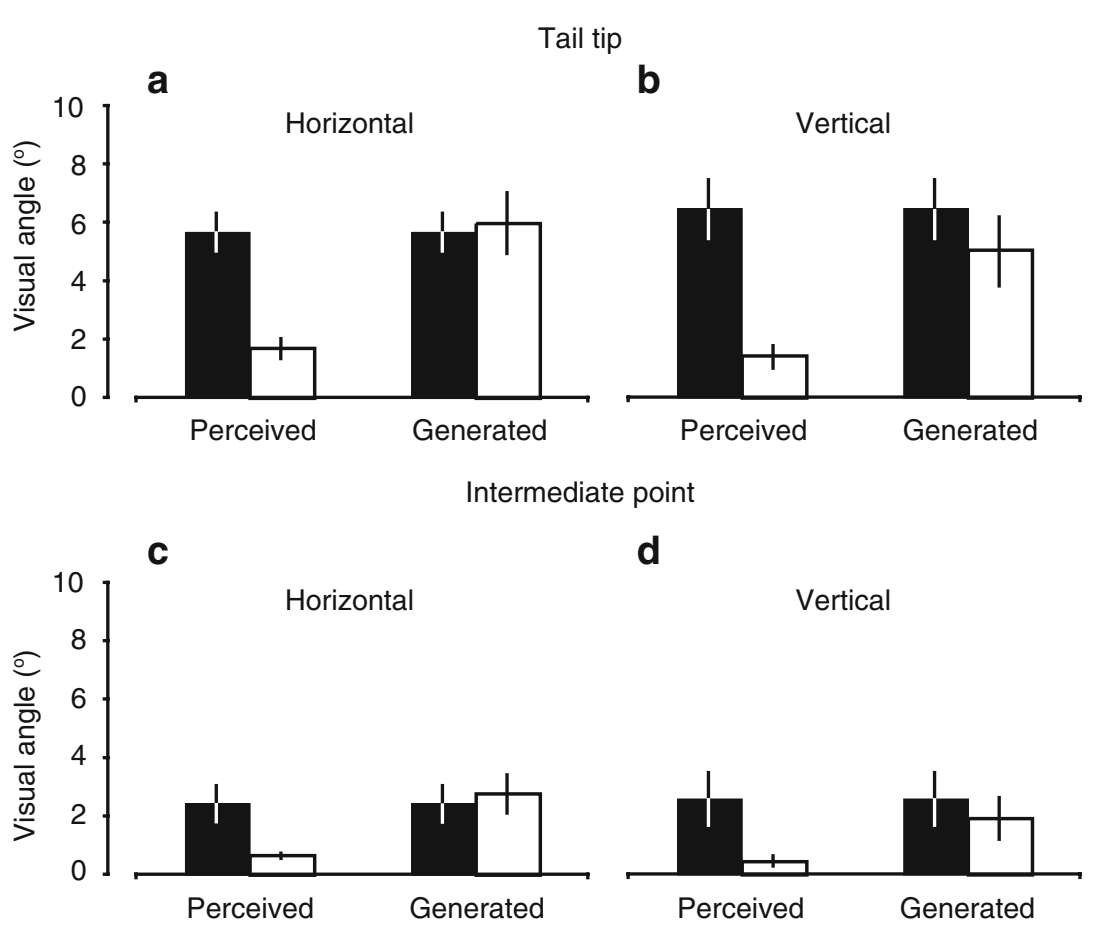

However, as above, signalling lizards did not significantly adjust signalling speeds to compensate for a likely reduction in perceived angular speeds (Fig. 5a, b, generated; Table 1). The effect size found for maximum angular speed achieved $(r=0.408)$ was the highest observed for the non-significant variables (Table 1).

\section{Discussion}

We compared $A$. muricatus tail flicking strategies in response to conspecific intruders and found no evidence to reject the null hypothesis of no difference between near (1 $\mathrm{m})$ and far $(3.5 \mathrm{~m})$ distance treatments (Table 1). To facilitate the interpretation of our results and comparison with our earlier study on plant motion effects, we have presented effect sizes (expressed as $r$ statistics) and associated $95 \%$ confidence intervals. For the temporal parameters we considered (duration, time in motion and effort), effect sizes less than 0.10 suggest that differences in nature are unlikely. A similar conclusion could be reached for variation in the spatial extent of the tail tip and average angular speeds (Table 1). The effect was a little stronger for variation in the spatial extent of the intermediate part of the tail. However, the effect for the stronger of the two (vertical) was in the opposite direction to that expected, showing a trend for near distances to be significantly greater than far distances. Of the variables we have considered, a medium effect size for maximum angular speed $(r=0.408)$ suggested that a significant outcome is
Table 2 Outcome of linear mixed effects models and corresponding effect sizes as perceived by the intruder

\begin{tabular}{|c|c|c|c|c|c|c|}
\hline & \multirow[t]{2}{*}{$F$ statistic } & \multirow[t]{2}{*}{$P$ value } & \multirow[t]{2}{*}{$d f$} & \multicolumn{3}{|c|}{ Effect size } \\
\hline & & & & $r$ & $95 \% \mathrm{CI}$ & \\
\hline \multicolumn{7}{|l|}{ Spatial } \\
\hline Tip_horizontal & 140.260 & 0.000 & 1,8 & -0.679 & -0.698 & -0.584 \\
\hline Tip-vertical & 63.937 & 0.000 & 1,8 & -0.906 & -0.958 & -0.675 \\
\hline Intermediate - horizontal & 18.125 & 0.004 & 1,8 & -0.751 & -0.885 & -0.319 \\
\hline Intermediate - vertical & 24.475 & 0.002 & 1,8 & -0.798 & -0.908 & -0.410 \\
\hline \multicolumn{7}{|l|}{ Angular speed } \\
\hline Maximum & 25.443 & 0.002 & 1,8 & -0.910 & -0.803 & -0.422 \\
\hline Average & 14.538 & 0.007 & 1,8 & -0.867 & -0.714 & -0.253 \\
\hline
\end{tabular}


a

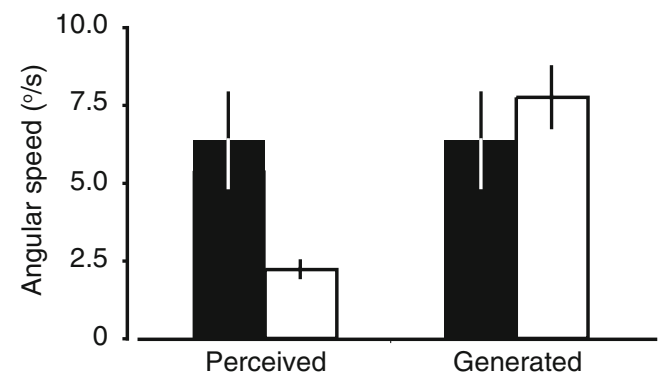

b

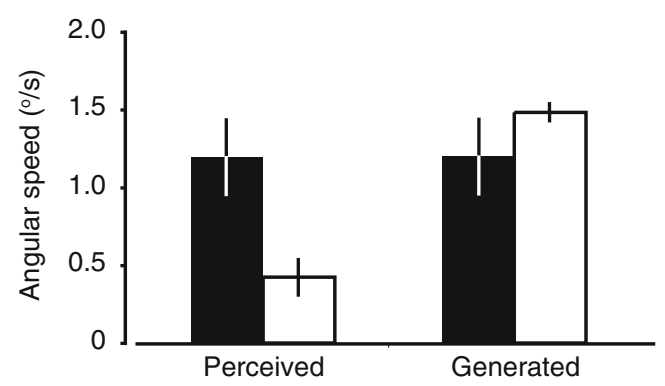

Fig. 5 Summary data for measurements of angular speed produced in response to near (closed square) and far (open square) intruders, shown separately for the perceived signal (distance $=1$ and $3.5 \mathrm{~m}$ ) and the generated signal (distance $=1 \mathrm{~m}$ for both). a Mean $( \pm \mathrm{SE}$ ) maximum angular speed across the full sequence. b Mean $( \pm \mathrm{SE})$ average angular speed across the full sequence

likely with a modest increase in sample size. Importantly, however, testing additional animals drawn from the same (normally distributed) population would not alter greatly the effect sizes calculated here. None of the effect size estimates in the present study came close to matching the effect size seen in the plant motion study $(r=0.701 ; 95 \% \mathrm{CI}$ $0.430-0.770$; calculated from the original data presented in Peters et al. 2007). The results of the present study and that of Peters et al. (2007), therefore, support a hypothesis that plant motion noise in the environment exerts a stronger effect on A. muricatus signalling behaviour than receiver distance per se.

Despite significant differences in the angular extent and angular speed at the eye of the receiver (Table 2), signals generated to intruders at near and far distances were equivalent (Table 1). In fiddler crabs $U$. perplexa, the spatial resolution of a single ommatidium (roughly $1^{\circ}$ ) probably necessitated changes in claw trajectories to ensure signals viewed from farther away were seen by multiple ommatidia (How et al. 2008). In contrast, the present dataset indicates that adjusting the spatial structure of tail flicking to compensate for sensory limitations in spatial resolution would be unnecessary. By quantifying this motor pattern in detail for the first time, we can deduce that tail flicks generated at near distances would remain within the sensitivity limits of receiver visual systems at even greater distances than that considered here. Although largely undetermined for lizards, the spatial resolution of A. muricatus visual systems is expected to be in the order of $0.2^{\circ}$ (estimated for Anolis auratus by Fleishman 1986), and as such, even the smallest signal amplitude observed here would remain detectable at a distance of $10 \mathrm{~m}(5 \mathrm{~cm}$ amplitude, $0.29^{\circ}$ visual angle). Signals of this amplitude generated outside of $10 \mathrm{~m}$ would certainly have reduced efficacy; however, consideration of signalling strategies at such distances would be irrelevant as they exceed typical signaller-receiver distances. Interactions between animals at such distances are unlikely due to the high probability of occluding objects in the cluttered environments these lizards inhabit. As lizards in the present study were found to generate signals at near distances that could, in principle, be detected at much greater distances, we speculate that tail flicking by $A$. muricatus is a low cost strategy for generating amplitudes sufficient for detection at a range of distances, thereby avoiding potential issues with spatial resolution. We are currently exploring the relative signalling costs (energetic, visibility to predators) of different motor patterns that comprise the A. muricatus display.

Although the effect size estimates for adjustments to movement speeds suggested that we cannot rule it out completely, our findings contrast with the facultative addition of an alerting component by the lizard A. gundlachi when signalling to distant receivers in adverse conditions (Ord and Stamps 2008). The additional display component used by $A$. gundlachi featured four-legged pushups characterised by fast speeds and high accelerations, which appear to differ markedly from the speciestypical head-bobbing that follows (Ord and Stamps 2008; quantitative detail of angular speeds not reported). As previously mentioned, however, the observed distance effect in their study is difficult to tease apart from other environmental factors that created adverse signalling conditions. Faster signalling may in fact be a strategy to overcome these constraints rather than compensating for receiver distances. Indeed, neural network modelling predicts that faster motion display speeds should improve signal detection among strong plant motion noise (Peters 2009); however, this prediction only holds when signals are performed closer to the receiver than the surrounding plants. The trend towards faster signalling in the present study is intriguing and it is possible that a combination of greater viewing distances and increased plant motion noise may bring out the predicted efficacy advantage of faster speeds. However, it is also unclear whether such a strategy 
would represent an advantage to $A$. muricatus as the segmentation of a thin tail might actually become more difficult at faster speeds. Neural network modelling again suggested that faster speeds would be futile when signalling at the same depth plane as wind-blown plants (Peters 2009), as is typical of these lizards.

Environmental plant motion noise and receiver distance are two theoretically important factors affecting the design and evolution of movement-based animal signals. Complementary studies have now considered each in isolation (Table 3). In calm conditions with no plant motion, the introductory component of $A$. muricatus displays are unaffected by receiver distance (this study). Conversely, variation in plant motion conditions significantly influenced introductory tail flicking strategies even when receivers were relatively close (Peters et al. 2007). In order to understand the factors that affect signal design in more detail, it will be important to compare signalling strategies in untested scenarios (Table 3; dotted lines). It is possible that the effect of receiver distance is only apparent when wind-blown plants generate motion noise that might affect detection. Playback studies with $A$. muricatus incorporating three-dimensional animations presented on video monitors (Peters and Evans 2007), as well as mechanised models (Peters 2008), have demonstrated that the detection of distant signals by receivers is delayed compared to closer signals in the presence of considerable plant motion. We predict therefore that lizards might seek to modify signal structure to compensate for receiver distances in this context. Consideration of the influence on signalling strategies in calm and windy conditions when receivers are farther away is also required. Response times in windy conditions are longer for more distant tail flicks (Peters 2008), which would also indicate a need to adjust signalling strategies.

Movement-based signalling strategies are likely to be influenced by a variety of factors in addition to plant motion and receiver distances. The influence of spacing patterns in a given microhabitat, particularly the relative distance between signaller and plants, have at least theoretically important implications for signalling strategies (Peters et al. 2008; Peters 2009). We also have to factor in constraints imposed by energetic limitations and costs associated with conspicuousness to predators (e.g. Allen et al. 2009). Predictably, exaggerated signals should occur either when the potential costs to the signaller are small, or in situations where they significantly increase efficacy. When the relative influence of all contributing factors is known, it should be possible to generate an efficacy/fitness function for a given movement-based visual signal, as has been achieved for acoustic signals (e.g. Brooks et al. 2005).

Acknowledgements Thanks to Elizabeth Allen and Don Sinclair for hosting us during animal collection and Robin and Steven Teding van Berkout for onsite support during the experiment. Thanks also to Tobias Merkle, Jochen Zeil and two anonymous referees for comments on an earlier draft, as well as the Neuroethology group at The Australian National University for constructive feedback. The Australian National University's Animal Experimentation Ethics Committee and the New South Wales National Parks and Wildlife Service approved all housing and experimental procedures. This work was funded by the Australian Research Council (DP0557018) and was hosted by the Edith and Joy London Foundation, Kioloa, NSW, Australia.

Table 3 Environmental wind conditions, receiver distance and tail flicking by A. muricatus

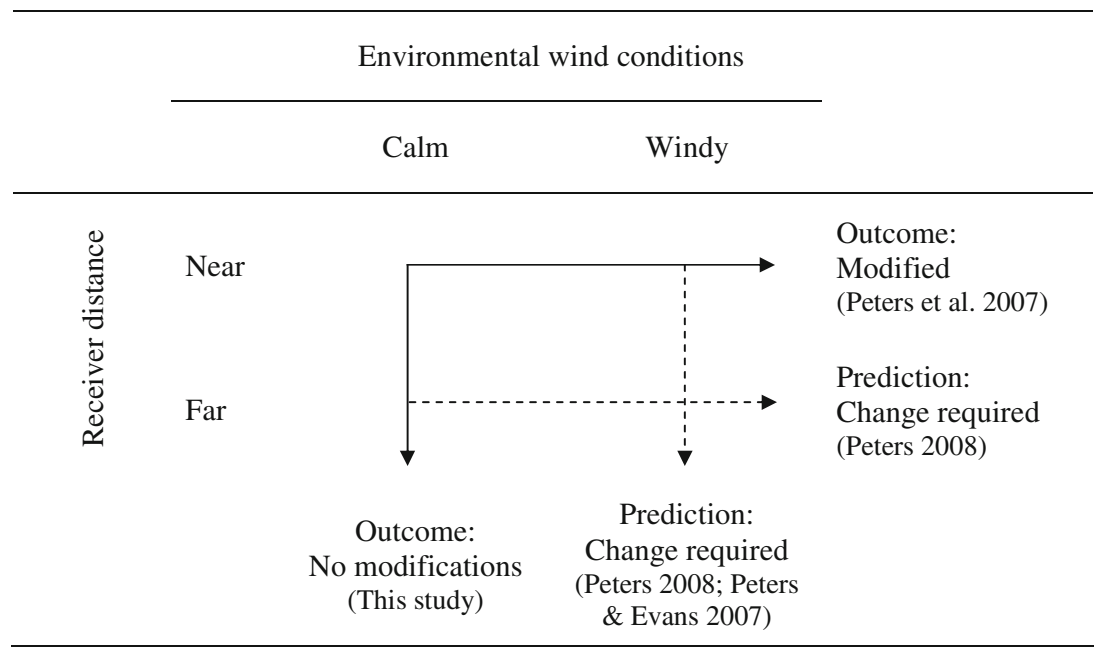




\section{References}

Allen E, Sinclair D, Allen S, Peters RA (2009) Amphibolurus muricatus (Jacky lizard). Avian predation. Herpetol Rev (in press)

Bradbury JW, Vehrencamp SL (1998) Principles of animal communication. Sinauer, Sunderland, MA

Brooks R, Hunt J, Blows M, Smith M, Bussiere L, Jennions MD (2005) Experimental evidence for multivariate stabilizing sexual selection. Evolution 59:871-888

Brumm H, Slater P (2006) Animals can vary signal amplitude with receiver distance: evidence from zebra finch song. Anim Behav 72:699-705

Couldridge V, van Staaden M (2004) Habitat-dependent transmission of male advertisement calls in bladder grasshoppers (Orthoptera; Pneumoridae). J Exp Biol 207:2777-2786

de la Torre S, Snowdon CT (2002) Environmental correlates of vocal communication of wild pygmy marmosets, Cebuella pygmaea. Anim Behav 63:847-856

Dusenbery DB (1992) Sensory ecology: how organisms acquire and respond to information. Freeman, New York, NY

Fleishman LJ (1986) Motion detection in the presence or absence of background motion in an Anolis lizard. J Comp Physiol A 159:711-720

How M, Hemmi JM, Zeil J, Peters RA (2008) Claw waving display changes with receiver distance in fiddler crabs, Uca perplexa. Anim Behav 75:1015-1022

Leal M, Fleishman LJ (2002) Evidence for habitat partitioning based on adaptation to environmental light in a pair of sympatric lizard species. Proc R Soc Lond B 269:351-359

Marten K, Marler P (1977) Sound transmission and its significance for animal vocalisations: I. Temperate habitats. Behav Ecol Sociobiol 2:271-290

Marten K, Quine D, Marler P (1977) Sound transmission and its significance for animal vocalisations: II. Tropical forest habitats. Behav Ecol Sociobiol 2:291-302

Nakagawa S, Cuthill IC (2007) Effect size, confidence interval and statistical significance: a practical guide for biologists. Biol Rev $82: 592-605$

New STD (2008) Vision in the sleepy lizard (Tiliqua rugosa): retinal organisation, visual acuity and ecological constraints. MSc. thesis, Flinders University, p 130

Ord T, Peters R, Clucas B, Stamps J (2007) Lizards speed up visual displays in noisy motion habitats. Proc R Soc Lond B 274:1057-1062

Ord T, Stamps JA (2008) Alert signals enhance animal communication in "noisy" environments. Proc Natl Acad Sci USA 105:1883018835
Owen P, Gordon N (2005) The effect of perceived intruder proximity and resident body size on the aggressive responses of male green frogs, Rana clamitans (Anura: Ranidae). Behav Ecol Sociobiol 60:736-741

Palombit R (1992) A preliminary study of vocal communication in wild long-tailed macaques (Macaca fasicularis). 2. Potential of calls to regulate intragroup spacing. Int J Primatol 13:183-207

Peters R (2008) Environmental motion delays the detection of movement-based signals. Biol Lett 4:2-5

Peters R (2009) Movement-based signalling and the physical world: modelling the changing perceptual task for receivers. In: Tosh $\mathrm{C}$, Ruxton $\mathrm{G}$ (eds) Modelling perception with artificial neural networks. Cambridge University Press, Cambridge, UK (in press)

Peters R, Evans C (2007) Active space of a movement-based signal: response to the Jacky dragon (Amphibolurus muricatus) display is sensitive to distance, but independent of orientation. J Exp Biol 210:395-402

Peters RA, Ord TJ (2003) Display response of the Jacky dragon, Amphibolurus muricatus (Lacertilia: Agamidae), to intruders: a semi-Markovian process. Austral Ecol 28:499-506

Peters RA, Clifford CWG, Evans CS (2002) Measuring the structure of dynamic visual signals. Anim Behav 64:131-146

Peters R, Hemmi J, Zeil J (2007) Signalling against the wind: modifying motion signal structure in response to increased noise. Curr Biol 17:1231-1234

Peters R, Hemmi JM, Zeil J (2008) Image motion environments: background noise for movement-based animal signals. J Comp Physiol A 194:441-456

R Development Core Team (2006) R: a language for statistical computing, Vienna. R Foundation for Statistical Computing, Vienna

Richards D, Wiley RH (1980) Reverberations and amplitude fluctuations in the propagation of sound in a forest: implications for animal communication. Am Nat 115:381-399

Römer H (1993) Environmental and biological constraints for the evolution of long-range signalling and hearing in acoustic insects. Philos Trans R Soc Lond B 340:179-185

Stamps JA, Barlow GW (1973) Variation and stereotypy in the displays of Anolis aeneus (Sauria: Iguanidae). Behaviour 47:67-94

Uy JAC, Endler JA (2004) Modification of the visual background increases the conspicuousness of golden-collared manakin displays. Behav Ecol 15:1003-1010

Wiley RH, Richards DG (1982) Adaptations for acoustic communication in birds: sound transmission and signal detection. In: Kroodsma DE, Miller EH (eds) Acoustic communication in birds, volume 1: production, perception, and design features of sounds. Academic, New York, pp 131-181 